\title{
A Parallel Implementation for Determining Genomic Distances Under Deletion and Insertion
}

\author{
Vijaya Smitha Kolli ${ }^{1}$, Hui Liu ${ }^{1}$, Michelle Hong Pan ${ }^{2}$, and Yi Pan ${ }^{1}$ \\ ${ }^{1}$ Department of Computer Science, Georgia State University, \\ Atlanta, GA 30303, USA \\ hui.anitaliu@gmail.com, pan@cs.gsu.edu \\ ${ }^{2}$ Centers for Disease Control and Prevention, Office of Workforce \\ and Career Development, Career Development Division, \\ Public Health Informatics Fellow Program, 1600 Clifton Rd, \\ Atlanta, GA 30333, USA \\ hdp1@cdc.gov
}

\begin{abstract}
As the need for comparing genomes of different species has grown dramatically with the fast progress of the Human Genome Project, the evolution at the level of whole genomes has attracted more and more attention from both biologists and computer scientists. They are especially interested in the scenarios in which the genome evolves through insertions, deletions, and movements of genes along its chromosomes. Marron et al proposed a polynomial-time approximation algorithm to compute (near) minimum edit distances under inversions, deletions, and unrestricted insertions. Our work is based on their algorithm, which carries out lots of comparisons and sorting to calculate the edit distance. These comparisons and sorting are extremely timeconsuming, and they result in decrease of computational efficiency. We believe the time of the algorithm can be improved through parallelization. We parallelize their algorithm via OpenMP using Intel C++ compiler for Linux 7.1, and compare three levels of parallelism: coarse grain, fine grain and combination of both. The experiments are conducted for a varying number of threads and different lengths of the gene sequences. The experimental results show that either coarse grain parallelism or fine grain parallelism alone does not improve the performance of the algorithm very much. However, the use of combination of both fine grain and coarse grain parallelism improves the performance of the algorithm drastically.
\end{abstract}

\section{Introduction}

A gene is the fundamental physical and functional unit of heredity. Each chromosome can be represented by an ordering of signed genes. The gene orders can be rearranged via evolutionary events like inversions and transpositions. The motivation of studying the gene sequencing arises in molecular biology. The ability to compare genomes of different species has grown considerably with the rapid advancement of Human Genome Project. One of the most effective methods of finding the similarity between genomes is to compare the order of appearance of identical genes in the two species. 
By finding the corresponding shortest edit distance between two signed gene permutations, we can know the smallest number of insertions, deletions, and inversions required to change one string of genes into another, where insertion, deletion and inversion are the process of adding, deleting and reversing a single or sequence of genes in the existing gene sequence respectively. However, it is very difficult to compute the edit distance between two genomes; for example, this problem is NP-hard for unsigned permutations even with equal gene content and only inversion allowed.

Many researchers have proposed various algorithms on finding the minimum edit distances $[3,4,5,6,10]$. Most of these algorithms involve lots of comparisons and sorting while computing edit distances. Marron et al's algorithm [2] is of particular interest for our implementation purposes. This algorithm handles duplications as well as insertions and presents an alternate framework for computing (near) minimal edit sequences involving insertions, deletions, and inversions.

Marron et al's algorithm is implemented sequentially, which is not efficient in terms of computation time, because the algorithm carries out many comparisons and sorting. Parallelism can be employed in the time-consuming comparisons and sorting, thus increasing the efficiency of the algorithm. We have performed profiling on the whole algorithm and identified the functions that are utilizing maximum time. We use OpenMP on Intel ${ }^{\circledR} \mathrm{C}++$ for Linux compiler 7.1 to parallelize the algorithm [9].

The rest of this paper proceeds as follows. Section 2 provides preliminaries on the sequential algorithm by Marron et al. We illustrate the details of our parallel implementation in Section 3. The experimental results are analyzed in Section 4. Section 5 draws the conclusion and proposes future works.

\section{Preliminary Existing Algorithm}

Marron et al's algorithm [2] is based on a new canonical form for edit sequences. They showed that shortest edit sequences can be transformed into equivalent sequences of equal length in which all insertions are performed first, followed by all inversions, and then by all deletions. This canonical form allows taking advantage of El-Mabrouk's exact algorithm for inversions and deletions, which can be extended by finding the best possible prefix of insertions, producing an approximate solution with bounded error.

\subsection{Canonical Form}

Marron et al [2] have proved some positive results about shortest edit sequences. These results will enable to obtain a "canonical form" into which any shortest edit sequence can always be transformed without losing optimality. Marron et al has proved the following two theorems.

Theorem 1: One two substrings become correctly oriented, they remain correctly oriented. 
Theorem 2: All insertions can be done before all inversions and deletions in a Minimum Edit Sequence.

\subsection{Sequences Cover}

A group of substrings from the target should be determined such that every element in the source appears in one of those substrings. The goal of the job is to cover all the non-deleted target elements with one from the subject. A minimal cover is one that uses fewest substrings of the subject. At each step, we try to cover the target from the left to as far as right as possible with contiguous subsequences of the subject. At last, this method produces a minimal cover by a greedy algorithm. The cover bound is proved in theorem 3 .

Theorem 3: There exists a cover of at most $2|\pi|+1$ for a sequence of $S$.

Now we can summarize Marron et al's Genomic Distances algorithm [2] in the following three steps.

Step 1: Relocate insertions to obtain the canonical form of sequences;

Step 2: Resolve duplicates by finding the minimum cover through greedy method;

Step 3: Then run exact EI-Mabrouk algorithm on the inversions and deletions.

\section{Details of Parallel Implementation}

This section gives the details of the implementation steps carried out to parallelize the Marron et al's genomic distances algorithm.

\subsection{Profiling}

Profiling is a good procedure to determine the most time-consuming parts of the code. By profiling the sequential code, we obtain a flat profile. Flat profile consists of the percentage of time used to complete the particular function, number of calls made for the function, self milli seconds per call and total milli seconds per call. From this flat profile, we can deduce which function uses greater percentage of time and further which function consumes more total milli seconds per call. The gprof utility provides a fast and easy way to do procedural-level profiling of the code. We use gprof and focus on the identification of such expensive functions with respect to time, and acknowledge the code to be parallelized. So that, these functions may utilize less time and eventually good speedup can be obtained. We compile the code using -pg option and run the code as usual. Then the code will produce an output file named gmon.out, which can be analyzed for further purposes. 


\subsection{Porting the Code Between GNU 3.2 and Intel C++ Compiler 7.1 for Linux}

In order to parallelize the algorithm using OpenMP we need to compile the program on Intel C++ compiler because GNU 3.2 does not support OpenMP. There are some significant porting issues encountered while porting program from GNU 3.2 to Intel $\mathrm{C}++$ compiler 7.1. The header files in GNU 3.2 compiler are declared with extension .h as \#include $<$ stack.h $>$ but in Intel $\mathrm{C}++$ compiler it is declared without.$h$ extension as \#include <stack $>$.

The initialization of the hash_map variables is much different in GNU compiler and Intel C++ compiler. In GNU, the hash_map is initialized with three parameters, while in Intel $\mathrm{C}++$ compiler it does not accept three parameters in the initialization. Due to this, the code has to be changed to compile successfully on the Intel C++ Compiler without the third parameter.

\subsection{Parallel Implementation of Genomic Distances Algorithm}

Genomic Distances algorithm is parallelized by using OpenMP on Intel C++ compiler 7.1 for Linux. Parallelism is implemented by applying fine-grain parallelism, coarsegrain parallelism and combination of both. OpenMP method makes use of fork-join technique. Master thread spawns team of threads as needed.

In coarse-grain parallelism, each function is given attention to find the functional dependencies, which is needed for synchronization, and mutual exclusive sections. Then the independent functions are taken and parallelized using the parallel and section directive. The mutually exclusive sections are provided with critical section directive, which specifies a region of code that must be executed by only one thread at a time.

In order to implement fine grain parallelism, first data dependency and critical sections within loops is checked to determine whether variables in nested loops should be declared as private or shared in OpenMP program after profiling and porting the program. The variables in the for loops are limited to individual threads, therefore they should be declared as private using private directive. Every thread has its own copy of these variables in parallel loops. A variable without the declaration is treated as shared variables by default. Second, the appropriate directive parallel for is added in front of loops to inform compilers to execute the code in parallel mode. All threads are scheduled dynamically with varying chunk sizes.

To implement the combination of coarse-grain and fine-grain parallelism in the Genomic Distances algorithm, both the parallel section and parallel for directive are applied appropriately in the required regions. Special attention is paid to mutual exclusive section.

\section{Analysis of Experimental Results}

This section discusses the experimental environment and results obtained in implementation of Genomic Distances algorithm via OpenMP. The Algorithm is 
parallelized by using fine-grain, coarse-grain and combination of both coarse and fine grain parallelism.

\subsection{Experimental Environment}

We test OpenMP performance by Intel $\mathrm{C}++$ compiler 7.1. This compiler has advanced optimization techniques for the Intel processor. The machine used is the Dell poweredge 6600 server and it has four quad SMP CPU's. Dell server is a powerful scalable parallel system. Experiments are conducted by varying the number of threads $(2,4,6,8)$ and length of gene sequences $(400,800,1000,2500,5000,10000)$. Speedup and program execution time for OpenMP are measured. Experiment results are verified and made sure that the identical gene sequences are utilized for sequential and parallel program.

\subsection{Coarse-Grain Parallelism}

As a first step to improve the efficiency of the Genomic Distance algorithm, we adopt OpenMP parallel sections construct for all the time-consuming functions. Special attention is paid to the functional dependencies. Fig. 1 compares sequential timing with the coarse-grain parallelism timing with varying the number of threads and length of gene sequences.

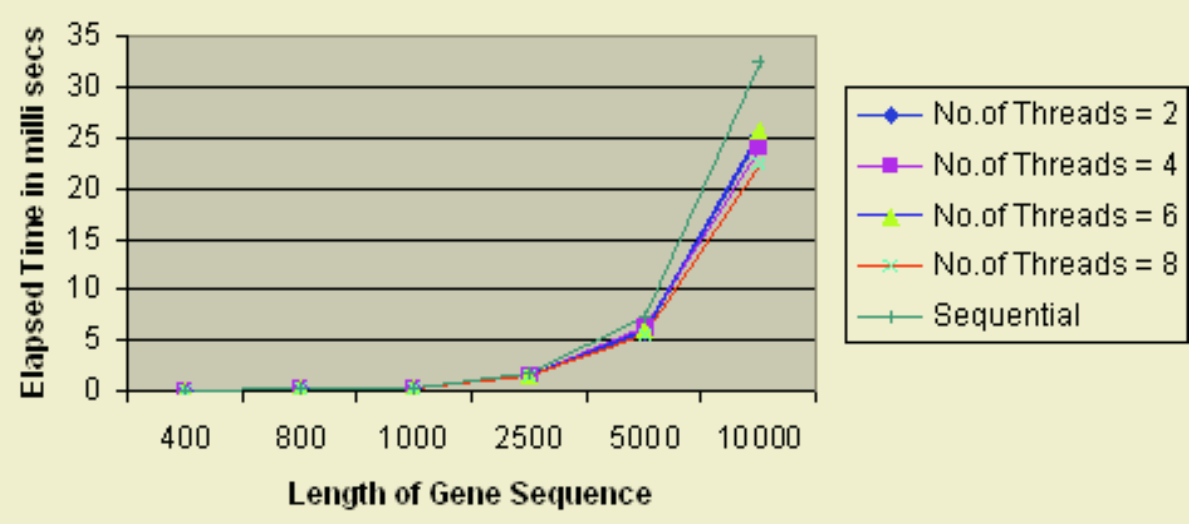

Fig. 1. Average execution time relative to the length of gene sequences under coarse-grain parallelism

Here the sequential algorithm was the most expensive in terms of execution time. We will compare the speedup values among three cases; coarse-grain parallelism, fine-grain parallelism, and the combination of coarse-grain and fine-grain parallelism when the length of gene sequence is 10000 , and the number of threads is 8 . For coarse-grain parallelism, the speedup value is 1.32 . Thus, we conclude that by parallelizing the algorithm with the parallel section construct there is not much 
improvement in execution time. Since there is a lot of dependency in the code, coarsegrain parallelism by sections does not yield a great performance progress.

\subsection{Fine-Grain Parallelism}

Later, to improve the efficiency of the Genomic Distance algorithm, we use OpenMP parallel for construct for all the time-consuming loops in the program. As the for directive specifies that the iterations of the loop immediately following it must be executed in parallel by the team of threads, each for loop will divide the array used into chunks of the specified size dynamically, and the number of threads will work on each individual chunk. With varying sequence size, care is taken to make the chunk size equally distributable among the available threads. Fig. 2 shows the results obtained as a part of fine-grain parallelism.

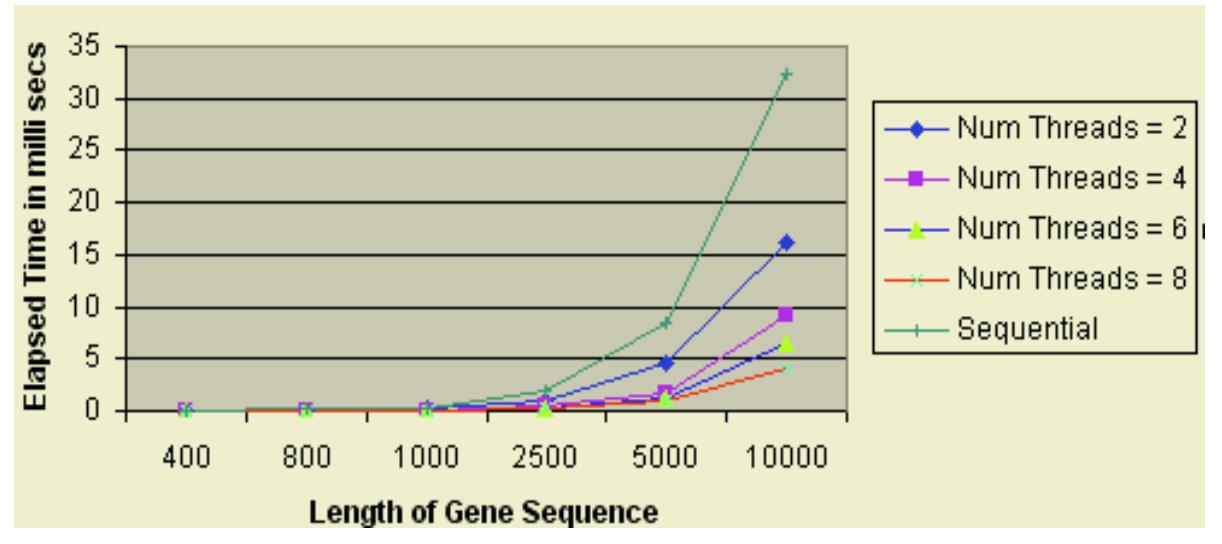

Fig. 2. Average execution time relative to the length of gene sequences under fine-grain parallelism

Here the sequential algorithm is the most consuming in terms of execution time. However, by parallelizing the algorithm with the parallel for construct there is a certain improvement. For example, the speedup value is 5.91 when gene sequence length is 10000 , and the number of treads is 8 . This is a good improvement compared with only coarse-grain parallelism.

\subsection{The Combination of Coarse-Grain Parallelism and Fine-Grain Parallelism}

Finally, to get a more efficient Genomic Distance algorithm, we adopt both OpenMP fine-grain and coarse-grain parallelism by implementing both parallel for construct and parallel sections construct to all the time consuming loops and functions. Fig. 3 shows the results obtained as a part of both fine-grain and coarse-grain parallelism. 


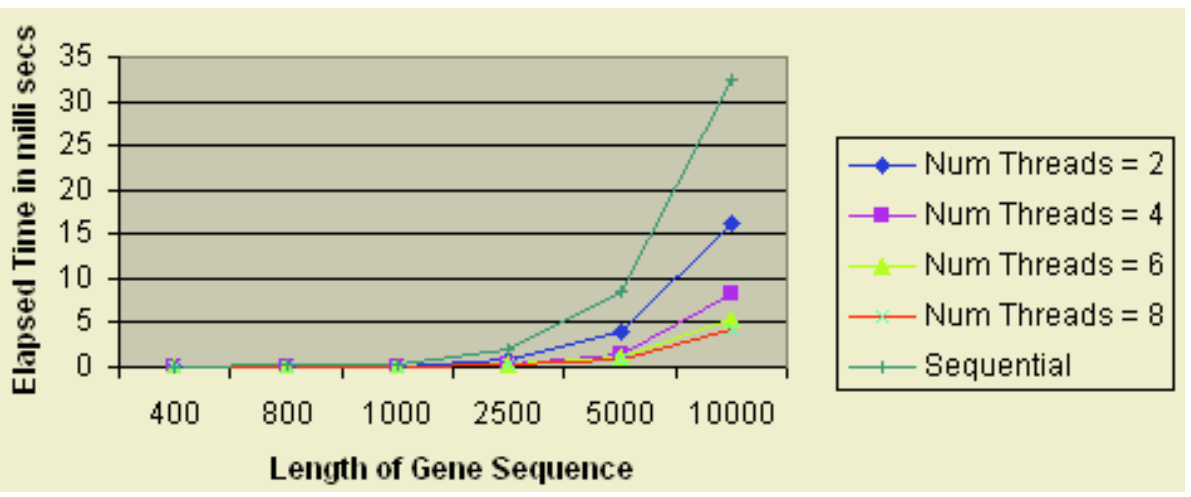

Fig. 3. Average execution time relative to the length of gene sequences under the combination of coarse-grain parallelism and fine-grain parallelism

As in the earlier cases, the figure shows that the sequential algorithm is the most consuming in terms of execution time. On the other hand, by parallelizing the algorithm with the parallel for and parallel sections constructs there is a very good improvement. For example, the speedup is 7.36 with gene sequence length 10000 and eight threads, which has achieved much better performance compared with only coarse-grain parallelism and only fine-grain parallelism.

By observing all the above three cases, we notice that the combination of both finegrain and coarse-grain parallelism has improved the performance of the algorithm to a great extent, because it implements both loop level parallelism, which takes care about all the time-consuming loops, and functional level parallelism, which handles the independent functions and execute them in parallel.

\section{Conclusion and Future Work}

The Genomic Distances algorithm in [2] provides a polynomial-time approximation algorithm with bounded error to compute edit distances under inversions, deletions, and unrestricted insertions from the perfectly sorted sequence to any other. The algorithm consists of many comparisons and sorting, so it is extremely timeconsuming. In order to improve the efficiency of the algorithm, we parallelize the algorithm using OpenMP. Furthermore, we study extensively the performance metrics for fine-grain and coarse-grain parallelism and both together. Since we can solve the problem quickly, we can handle larger gene sequences which are very important in practices in bioinformatics research.

From our experimental results, we conclude that coarse grain parallelism is not effective for this algorithm since there is lot of functional dependencies, and many functions are not able to execute concurrently. There is improvement in performance when the algorithm is parallelized with fine grain parallelism, for all the timeconsuming for loops are made to run in parallel. When the algorithm is parallelized by 
the combination of both fine and coarse grain parallelism, there is very good improvement in the efficiency of the algorithm.

In the future, we can obtain better performance of the Genomic Distances algorithm by using both MPI and OpenMP. MPI handles the larger-grained communications among multiprocessors, while the lighter-weight threads of OpenMP handle the processor interactions within each multiprocessor. By adding MPI function calls to the OpenMP source program, the program can be transformed into a MPI/OpenMP program suitable for execution on a cluster of multiprocessors.

\section{References}

1. D.A. Bader, B.M.E. Moret, and M. Yan. A fast linear-time algorithm for inversion distance with an experimental comparison. J. Comput. Biol., 8(5):483. 491, 2001.

2. Mark Marron, Krister M. Sweson, and Bernard M. E. Moret. Genomic Distances under Deletions and Insertions. Proc. 9th Int'l Combinatorics and Computing Conf. COCOON'03, 2003, 537-547.

3. A. Caprara. Sorting by reversals is difficult. In Proc. 1st Int'l Conf. on Comput.Mol. Biol. RECOMB97, pages 75.83. ACM Press, 1997.

4. S. Hannenhalli and P. Pevzner. Transforming cabbage into turnip (polynomial algorithm for sorting signed permutations by reversals). In Proc. 27th Ann.Symp. Theory of Computing STOC 95, pages 178.189. ACM Press, 1995.

5. N. El-Mabrouk. Genome rearrangement by reversals and insertions/deletions of contiguous segments. In Proc. 11th Ann. Symp. Combin. Pattern MatchingCPM 00, volume 1848 of Lecture Notes in Computer Science, pages 222.234. Springer-Verlag, 2000.

6. T. Liu, B.M.E. Moret, and D.A. Bader. An exact linear-time algorithm for computing genomic distances under inversions and deletions U. New Mexico, TR-CS-2003-31.

7. Tutorial on OpenMP, http://www.llnl.gov/computing/tutorials/openMP/

8. Michael Quinn, Parallel Programming in $C$ with MPI and OpenMP, The McGraw-Hill Companies, 2004.

9. Intel C++ Compiler for Linux, http://www.intel.com/software/products/compilers/clin/ clinux.htm

10. Haim Kaplan, Ron Shamir and Robert E. Tarjan, "Faster and Simpler Algorithm for Sorting Signed Permutations by Reversals", Proc. SODA 97 pages 344-351,SIAM Journal on Computing 29 (3) 880--892 (1999). 